\title{
Avaliação das condições de saúde bucal de pessoas com fissuras labiopalatinas em Belém, norte do Brasil
}

\author{
Evaluation of oral health conditions in people with cleft lip and palate in Belém, northern \\ Brazil
}

\section{Evaluación de las condiciones de salud bucal de personas con labiopalatinas claras en belém, norte de Brasil.}

Pedro Henrique Noronha Cavalcante ${ }^{1 *}$, Gabriel Henrique Soares Cavalcante ${ }^{1}$, Ricardo Roberto de Souza Fonseca ${ }^{1}$, Tabata Resque Beckmann Carvalho², Silvio Augusto Fernandes de Menezes², Paula Mendes Acatauassú Carneiro², Marcelo Newton Carneiro², Michel Wagner de Souza Matos², Gyselle Ribeiro De Carvalho Oliveira ${ }^{1}$, Tatiany Oliveira De Alencar Menezes ${ }^{1}$.

\section{RESUMO}

Objetivo: Avaliar as condições de saúde bucal de pessoas com fissura labiopalatinas (PFLP) atendidos em um centro especializado em Belém-PA. Métodos: Pesquisa de caráter transversal com análises descritiva, quantitativa e qualitativa na Associação de Apoio ao Fissurado Labiopalatal Sorrisos Largos (AAFLSL), pelo exame clínico da cavidade oral e registros de prontuários de 29 associados PFLP, de todas as idades e ambos os sexos, cadastrados na (AAFLSL). Levou-se em consideração: Presença de cárie, prevalência dos tipos de fissuras, presença de hábitos deletérios, período de internação e reparação cirúrgica das fissuras. Os dados foram calculados por frequência absoluta e relativa no Microsoft excel®. Resultados: Das PFLP avaliadas; houve prevalência do sexo masculino. $62 \%$ estão livres de cárie. A fissura transforame unilateral completa demonstrou maior incidência. Cerca de $62 \%$ possuem algum hábito deletério. $83 \%$ passaram por período de internação nos 2 últimos anos. Quanto as cirurgias reparadoras, cerca de $82 \%$ já passaram pela queiloplastia e 62\% passaram pela palatoplastia Conclusão: Os dados obtidos mostram condições satisfatórias de saúde bucal das pessoas analisadas, entretanto a presença de hábitos parafuncionais é um fator de risco à saúde. Por fim, conhecer o perfil do paciente fissurado é essencial para que sejam estabelecidos protocolos de tratamentos.

Palavras-chave: Fenda labial, Cárie dentária, Pessoas com deficiência.

\begin{abstract}
Objective: To evaluate the oral health conditions of people with cleft lip and palate attended in a specialized center in Belém. Methods: This is a cross-sectional study with descriptive, quantitative and qualitative analysis. The research was developed at the Association for Support to the Cleft Labiopalatal Sorrisos Largos (AAFLSL), located in the city of Belém-PA. The data were obtained from an oral cavity clinical exam and medical records. The data obtained were tabulated in an excel spreadsheet. Results: From the analysis of 29 fissured individuals from 1 to 24 years old, it was found that males were the most frequent, in addition to a greater participation of children aged 1 to 3 years. The analysis of the presence of caries in relation to age showed that more than $50 \%$ of those analyzed did not have caries. The complete unilateral transforamen cleft was the one with the highest incidence. Conclusion: The data obtained show satisfactory oral health conditions of the people analyzed, however the presence of parafunctional habits is a risk factor for health. Finally, knowing the profile of the cleft patient is essential for establishing treatment protocols.
\end{abstract}

Keywords: Cleft lip, Dental caries, People with disabilities.

1 Universidade Federal Do Pará (UFPA), Belém - PA. *E-mail: pedro25henrique10@gmail.com

${ }^{2}$ Centro Universitário Do Estado Do Pará (CESUPA), Belém - PA. 


\section{RESUMEN}

Objetivo: Evaluar las condiciones de salud bucal de personas con labio leporino y paladar hendido en una asociación en Belém, destacando la prevalencia de hendiduras. Métodos: Se trata de un estudio transversal con análisis descriptivo, cuantitativo y cualitativo. La investigación se desarrolló en la Asociación de Apoyo a la Hendidura Labiopalatal Sorrisos Largos (AAFLSL), ubicada en la ciudad de Belém-PA. Los datos se obtuvieron del examen clínico de la cavidad bucal de las hendiduras y se registraron en las historias clínicas. Los datos obtenidos se tabularon en una hoja de cálculo de Excel. Resultados: Del análisis de 29 individuos fisurados de 1 a 24 años, se encontró que los varones fueron los más frecuentes, además de una mayor participación de los niños de 1 a 3 años. El análisis de la presencia de caries en relación a la edad mostró que más del $50 \%$ de los analizados no tenían caries. La hendidura transforamen unilateral completa fue la de mayor incidencia. Conclusión: Los datos obtenidos muestran condiciones de salud bucal satisfactorias de las personas analizadas, sin embargo la presencia de hábitos parafuncionales es un factor de riesgo para la salud. Finalmente, conocer el perfil del paciente con hendidura es fundamental para establecer protocolos de tratamiento.

Palabra clave: Labio leporino, Caries dental, Personas con discapacidad.

\section{INTRODUÇÃO}

As fissuras labiopalatinas (FLP) consistem em anomalias de má formação congênitas de lábio e palato que se dão por uma abertura/ruptura nessas regiões. O processo de formação da face e da cavidade oral são de natureza bastante complexa e envolve o desenvolvimento de múltiplos processos teciduais, devendo se unir e fusionar de modo extremamente ordenado. Distúrbios no crescimento desses processos ou nas suas fusões podem resultar na formação de fendas orofaciais (MONE F, et al., 2020; SILVA YC, et al., 2018; KUHN VD, et al., 2016). A formação dessas fendas se dá pela não fusão dos processos palatinos e nasais mediais durante o desenvolvimento embriológico, entre a quarta e oitava semana de gestação (Amorim et al., 2019; Pires et al., 2020).

As FLP estão entre as malformações faciais congênitas mais comuns (MONE F, et al., 2020; SILVA YC, et al., 2018). Além disso, há alguns fatores modificadores para prevalência de FLP que podem variar consideravelmente de acordo com: geografia, etnia, gênero, hábitos, hereditariedade, condições socioeconômicas e faixas etárias materna e paterna (KUHN VD, et al., 2016).

As FLP possuem uma alta incidência, entre os anos de 2005 a 2016, estima-se que a incidência no Brasil é de aproximadamente 0,51 a cada 1000 nascidos vivos. Quanto as taxas em cada região, no mesmo período, foi analisado que as regiões que registraram a maior incidência foram Sul e Sudeste apresentando taxas médias superiores à média brasileira $(0,72 / 1000$ e 0,54 / 1000 nascidos vivos, respectivamente) e as menores taxas médias foram observadas nas regiões Nordeste $(0,39$ / 1000 nascidos vivos) e Norte $(0,45$ / 1000 nascidos vivos). Por fim, o Centro-Oeste com taxa média de (0,5 / 1000 nascidos vivos (SHIBUKAWA BM, et al., 2019). Como mencionado anteriormente, essas variações de incidências estão associadas a diversos fatores que variam de acordo com as regiões analisadas (KUHN VD, et al., 2016).

A etiologia das FLP é multifatorial e está relacionada basicamente a fatores genéticos e ambientais. Fatores de risco para propensão ao desenvolvimento de fendas pode estar relacionada a um número maior ou menor de genes e fatores ambientais, como uso de álcool e drogas, idade, fumo, uso de medicamentos, deficiência nutricional, entre outros (COSTA VC, et al., 2019).

A ocorrência dessas FLP pode comprometer a saúde de um indivíduo em diferentes níveis, devido às implicações estéticas, funcionais e psicológicas e dependendo do tipo de fissura, há um menor ou maior comprometimento das estruturas como lábio e palato (BERNARDO B, et al., 2017; SILVA BJ, et al., 2018). O comprometimento estético pode ser significativo, pois além da ruptura lábio/palato é comum em FLP a ocorrência agenesias, supranumerários e alterações na forma da coroa dentária. Desta forma, interferem no processo de comunicação, podendo levar a prejuízos na interação social e na aprendizagem, assim como, no desenvolvimento de competências cognitivas e afetivas (BERNARDO B, et al., 2017; PESSOA EA, et al., 2017). 
A classificação mais utilizada para fissuras é a classificação de Spina, de acordo com essa classificação há três grandes grupos, possuindo como parâmetro a localização do forame incisivo: pré-forame, trasnforame e pós-forame. As fissuras transforame são as mais complexas e se estendem desde os lábios até o palato, comprometendo de forma mais significativa a dentição, fonação, audição e deglutição (BERNARDO B, et al., 2017; SILVA BJ, et al., 2018). A fissura transforame segundo diversos estudos é a de maior incidência comparada as pré-forame e pós-forame (KUHN VD, et al., 2016).

As crianças que nascem com FLP necessitam de constante suporte nutritivo, emocional e intelectual (MONE F, et al., 2020). As fissuras trazem limitações e complicações que expõem o paciente a um grande risco de desnutrir, principalmente pela sucção insuficiente, a deglutição excessiva de ar com reflexos nasais e engasgos, ao cansaço e ao gasto energético devido a alimentação demorada, tendo uma baixa ingestão de nutrientes (AMORIM SM, et al., 2019; SILVA YC, et al., 2018). Além disso, a presença de infecções pode ser recorrente, as crianças podem aspirar o alimento, provocando infecções como otites e pneumonias, uma vez, que nesses casos, há comunicação buconasal (KUHN VD, et al., 2016). Sob esse panorama da situação fica nítido que o tratamento de um fissurado requer uma equipe multidisciplinar, formada por enfermeiros, médicos, nutricionistas, fonoaudiólogos, cirurgiões-dentistas e psicólogos (KUHN VD, et al., 2016; SANTOS RC, et al., 2019).

O tratamento de um fissurado deve ser iniciado precocemente e pode durar muitos anos, ocorrendo em várias etapas e passando por diversos profissionais. Esses tratamentos podem ser divididos em não cirúrgicos e cirúrgicos. Dos tratamentos não cirúrgicos, o fonoaudiólogo estimula a movimentação peribucal e correta interposição da língua. De forma precoce, o cirurgião-dentista pode iniciar o tratamento ortodôntico e realizar o selamento de cicatrículas e fissuras, a fim de reduzir o risco à cárie. O acompanhamento psicológico também deve ser iniciado ainda na infância, pois o estigma negativo da fissura compromete o emocional de PFLP (SANTOS RC, et al., 2019).

Os tratamentos cirúrgicos podem incluir a queiloplastia (cirurgia reparativa dos lábios) que deve ser realizada por volta de 2 meses de idade e a palatoplastia (cirurgia reparativa do palato), devendo ser feita até os 2 anos de idade (ANDRADE CA, et al., 2019). A presença do cirurgião dentista é fundamental em todas as etapas do tratamento (OLIVEIRA MF e BANDEIRA MB, 2018; PESSOA EA, et al., 2017).

Logo essa pesquisa tem por objetivo fazer um levantamento sobre as condições de saúde bucal em crianças portadoras de FLP em uma associação do município de Belém-PA.

\section{MÉTODOS}

Esta pesquisa teve caráter transversal com análises descritiva, quantitativa e qualitativa. A pesquisa foi desenvolvida na Associação de Apoio ao Fissurado Labiopalatal Sorrisos Largos (AAFLSL), localizada no município de Belém-PA, no serviço direcionado aos pacientes e em dias já destinados ao atendimento clínico.

O trabalho foi submetido e aprovado no Comitê de Ética em Pesquisa com Seres Humanos do Instituto de Ciências da Saúde da Universidade Federal do Pará, obtendo o registro de autorização sob o n 3.280.658. Todos os participantes da pesquisa tiveram assegurados seus direitos de privacidade através do termo de consentimento livre e esclarecido (TCLE).

Dos 60 pacientes cadastrados e associados no programa de portadores de FL na AAFLSL, 29 pacientes aceitaram participar do estudo. Houve convite de participação aos responsáveis e os critérios de inclusão foram: PFLP regularmente inscritos na AAFLSL, independentemente da idade e do sexo, e que seus pais e/ou seus responsáveis autorizaram a participação por meio do Termo de Consentimento Livre e Esclarecido (TCLE). Após o preenchimento do TCLE foi realizada a coleta de dados a partir do exame físico. Os critérios de exclusão foram: PFLP que não assentiram a participação na pesquisa ou que foi recusada por seus responsáveis, fissurados que não aceitaram a realização do exame, PFLP que não residem em Belém e região metropolitana.

Para a coleta de dados houve colaboração de 2 discentes do curso de odontologia da Universidade Federal do Pará- UFPA. Esses avaliadores foram calibrados em exame intra-oral pela aplicação do teste Kappa. 
Inicialmente foram selecionados, de forma aleatória, 16 pacientes para participarem do teste de calibração com os 2 examinadores, ambos realizaram a avaliação clínica nesses pacientes. Passados 7 dias, uma nova avaliação foi feita para determinar a concordância intra e inter-examinador, e a aferição do teste Kappa para confiabilidade das análises com o uso do software IBM® SPSS® Statistics 20. O Kappa obtido para análise intra-examinador foi de 0.921 e para a análise de inter-examinadores foi de 0.812 .

A coleta de dados foi realizada a partir do exame clínico da cavidade oral de PFLP em um ambiente com iluminação indireta, por 2 discentes do curso de Odontologia, calibrados em exame intra-oral. Todos utilizaram protetores individuais, a fim de avaliar a condição bucal com auxílio de abaixador de língua e gaze. Os dados epidemiológicos e bucais avaliados foram: sexo, idade, presença de cárie por faixa etária, prevalência do tipo de fissuras, hábitos parafuncionais, histórico de internação e a situação cirúrgica. Os dados foram anotados em fichas clínicas previamente elaboradas e posteriormente, as informações foram repassadas para 0 programa Microsoft $\AA^{\circledR}$ Excel para avaliação das frequências relativas e absolutas das variáveis analisadas.

Devem descrever de forma clara e sem prolixidade as fontes de dados, a população estudada, a amostragem, os critérios de seleção, procedimentos analíticos e questões éticas relacionadas à aprovação do estudo por comitê de ética em pesquisa (pesquisa com seres humanos e animais) ou autorização institucional (levantamento de dados onde não há pesquisa direta com seres humanos ou animais).

\section{RESULTADOS}

Os dados deste artigo são advindos do projeto de extensão Saúde Bucal em Pacientes Portadores de Necessidades Especiais (SBPNE). Neste estudo, há presença de ambos os sexos, sem discrepância na proporção. A Faixa etária dos fissurados presentes no estudo, compreendeu de 1 a 24 anos de idade, sendo a média das idades corresponde a aproximadamente 4 anos de idade. No que compreende a classificação dos tipos de fissura, em todos os tipos houve pelo menos 1 fissurado em cada classificação, desta forma, nenhuma classe de fissura foi zerada. A presença de hábitos parafuncionais ocorreu de forma significativa, bem como o período de internação nos últimos 3 anos. Além disso, as cirurgias reparadoras de lábio e palato tem uma elevada incidência.

As variáveis epidemiológicas e bucais avaliadas foram descritas na tabela 1. O sexo masculino foi o de maior frequência com 55\%. Em relação a idade, crianças na faixa etária de 1-3 anos de idade foram a maioria, representando aproximadamente $60 \%$ dos fissurados, cerca de $52 \%$ dos participantes entre um a três anos estavam ausentes de cárie dentária.

Tabela 1 - Frequência Absoluta e Relativa (\%) da prevalência de sexo e presença de cárie em relação à idade, $n=29$. Belém-PA, 2021.

\begin{tabular}{ccc}
\hline VARIÁVEIS & $\mathbf{N}$ & $\%$ \\
\hline Sexo & & \\
\hline Masculino & 16 & 55 \\
Feminino & 13 & 45 \\
\hline Faixa etária & & 58,7 \\
\hline $1-3$ & 17 & 24,2 \\
$4-6$ & 7 & 10,3 \\
$7-9$ & 3 & 6,8 \\
\hline 10 & 2 & \\
\hline Presença de cárie por faixa etária (idade em anos) & & 6,8 \\
\hline $1-3$ & 2 & 17,3 \\
$4-6$ & 5 & 10,3 \\
$7-9$ & 3 & 3,5 \\
\hline
\end{tabular}

Fonte: Cavalcante PHN, et al., 2021. 
Como já mencionado as fissuras podem ser agrupadas em 3 classes: pré-forame, transforame e pósforame, logo segue os percentuais de cada grupo. As fissuras transforames (unilalateral e bilateral) representam juntas $76.2 \%$ das fissuras das pessoas avaliadas. Já as fissuras pré-forame (unilateral e bilateral) se somadas representam 13,6\%. Por fim, a classe pós-forame (incompleta e completa) se também forem somadas representam um valor de 10,2\%. O detalhamento de cada fissura específica segue na Tabela 2.

Tabela 2 - Prevalência dos tipos de fissuras com base na classificação de Spina, n=29. Belém-PA, 2021.

\begin{tabular}{ccc}
\hline Variável & N & $\%$ \\
\hline Tipos de fissura & 1 & 3,4 \\
Pré-forame unilateral incompleta & 1 & 3,4 \\
Pré-forame unilateral completa & 1 & 3,4 \\
Pré-forame bilateral incompleta & 1 & 3,4 \\
Pré-forame bilateral completa & 12 & 41,6 \\
Transforame unilateral completa & 10 & 34,6 \\
Transforame bilateral completa & 1 & 3,4 \\
Pós-forame incompleta & 2 & 6,8 \\
Pós-forame completa & & \\
\hline
\end{tabular}

Fonte: Cavalcante PHN, et al., 2021.

A Tabela 3 demonstra a frequência relativa dos hábitos parafuncionais. Notavelmente o hábito de ranger os dentes foi o mais frequente dos hábitos deletérios, representando quase $28 \%$ dos pacientes analisados.

Tabela 3 - Presença de hábitos parafuncionais, n=29. Belém-PA, 2021.

\begin{tabular}{ccc}
\hline Variável & $\mathbf{N}$ & $\%$ \\
\hline Hábitos & & 0 \\
Chupar dedo & 0 & 6,8 \\
Respiração bucal & 2 & 3,5 \\
Morder objetos & 1 & 27,6 \\
Ranger os dentes & 8 & 3,5 \\
Usar chupeta & 1 & 6,8 \\
Roer as unhas & 2 & 13,9 \\
Dois ou mais hábitos & 4 & 38 \\
Sem hábitos & 11 & \\
\hline
\end{tabular}

Fonte: Cavalcante PHN, et al., 2021.

Na Tabela 4 a avaliação do quesito período de internação nos últimos 3 anos também levou em consideração as internações durante as cirurgias reparadoras de lábio e palato, logo alguns fissurados que estiveram internados nos últimos 3 anos coincidiram também com a análise da realização de cirurgias reparadoras.

Tabela 4- Histórico de internação nos últimos 3 anos e situação cirúrgica, n=29. Belém-PA, 2021.

\begin{tabular}{ccc}
\hline Variáveis & N & $\%$ \\
\hline Internação & & 83 \\
\hline Esteve internado & 24 & 27 \\
\hline Não esteve internado & 5 & \\
\hline Situação Cirúrgica Queiloplastia & & 82,8 \\
\hline Já realizou & 24 & 6,9 \\
Ainda não realizou & 2 & 10,3 \\
Não necessita & 3 & \\
Palatoplastia & & 62,1 \\
Já realizou & 18 & 31,1 \\
Ainda não realizou & 9 & 6,8 \\
Não necessita & 2 &
\end{tabular}

Fonte: Cavalcante PHN, et al., 2021. 
Quanto a queiloplastia, esses 6,9\% que ainda não realizaram já estão com mais de 1 ano de idade, ou seja, já passaram do tempo ideal para o procedimento que é a partir dos 2 a 3 meses de vida. Sobre a palatoplastia, a cirurgia reparadora do palato, os dados mostram que cerca de $31,1 \%$ ainda não realizaram esse procedimento e dentro desse número dos que ainda não passaram pela palatoplastia, cerca de $23 \%$ já passaram da idade ideal de realização do procedimento, que é entre 1 e 2 anos de idade.

\section{DISCUSSÃO}

A literatura indica que a maior incidência de fissurados é no sexo masculino. (KUHN VD, et al., 2016; LACERDA RH, et al., 2012; MONE F, et al., 2020), o que vai de encontro ao resultado adquirido no estudo, no qual obteve-se que $55 \%$ dos participantes foram do sexo masculino e $45 \%$ do sexo feminino. Não há conhecimento do motivo dessa prevalência maior do sexo masculino, no entanto como foi mencionado, a ocorrência de FLP está associada a fatores ambientais e hereditários, havendo inúmeras variáveis a serem consideradas para que se chegue a incidência de uma fissura. Além disso, a prevalência muda de local para local e envolve fatores socioculturais (KUHN VD, et al., 2016; SILVA BJ, et al., 2018).

A cárie afeta grandemente a população infantil, sendo uma das doenças crônicas mais comuns da infância, por causar dor e sensibilidade afeta o ato de comer, o que interfere no ganho de peso e crescimento de uma criança. Além disso, distúrbios de sono são comuns, afetando o rendimento e concentração das crianças (NUNES VH e PEROSA GB, 2017). Pacientes fissurados, muitas vezes, apresentam elevado risco para cárie dental. Isso ocorre por diversas anomalias que podem estar associados à FLP, como dentes hipoplásicos ou aglomerados na região da fissura, logo pode ser entendida a maior prevalência de doença cárie nos pacientes fissurados em relação a não fissurados (ALVES BR, et al., 2019; ROCHA CL, 2018).

A higienização da cavidade oral de fissurados é um desafio para os responsáveis pela maior dificuldade em limpar a região da fissura. Mas como para qualquer pessoa a escovação é essencial. A ocorrência de cárie está associada a alimentação rica em sacarose e uma higienização ineficiente, além de fatores predisponentes como anomalias (ROCHA CL, 2018).

Nesse estudo foi obtido que crianças de 1 a 3 anos apresentam baixa incidência de cárie, $51,8 \%$ estavam livres de cáries e outros 6,8\% apresentavam algum dente cariado. Quando se avança na idade para maiores de 4 anos, pode-se notar que há um aumento na incidência de cárie, de 4 a 6 anos, 17,3\% apresentavam cárie e outros 6,8\% não apresentavam. Especificamente com relação à dentição decídua, apesar de dados de prevalência muito diversos, observa-se um aumento significativo de cárie à medida que aumenta a idade (NUNES VH e PEROSA GB, 2017). Pode-se relacionar a menor incidência de cárie em idades baixas a um maior cuidado dos responsáveis em realizar a higienização eficaz, porém com o avançar da idade os pais acabam naturalmente por reduzir esse cuidado, evidenciando a maior ocorrência da cárie.

Dependendo do tipo de FLP há um menor ou maior comprometimento tanto das funções fisiológicas quanto psicológicas. A fissura pode comprometer severamente a fala, a alimentação, o posicionamento dentário e a estética. Sem o devido tratamento, as fissuras podem provocar sequelas graves, como a perda da audição, problemas de fala e déficit nutricional, além do sofrimento com o preconceito (KUHN VD, et al., 2016; SILVA BJ, et al., 2018).

Com a classificação é possível se pensar em um diagnóstico, na reabilitação e no prognóstico (BERNARDO B, et al., 2017). A literatura aponta para uma maior prevalência das fissuras transforame, em que se há o envolvimento dos lábios e toda extensão do palato. Neste estudo foi obtido que $76,2 \%$ dos fissurados possuem a FL transforame, seja ela unilateral ou bilateral. Assim como outros estudos presentes na literatura (LACERDA RH, et al., 2012; MONE F, et al., 2020; SANTOS RC, et al., 2019; SILVA YC, et al., 2018).

As dificuldades começam logo após o nascimento e se estendem no decorrer da vida, as fendas que envolvem o palato prejudicam o poder de sucção, assim os recém-nascidos apresentam dificuldade de sugar o leite materno durante a amamentação devido a fraca pressão intraoral durante a realização dessa função (SILVA SM, et al., 2018). Mesmo sendo um processo difícil e demorado devido à pouca ingestão de leite, a amamentação é fundamental para o desenvolvimento da musculatura além das propriedades nutricionais e imunológicas (MONE F, et al., 2020). 
O paciente fissurado pode ter sua saúde agravada a partir da presença de hábitos parafuncionais. No presente estudo pode-se notar que uma grande parte dos avaliados apresenta algum vício/mania voluntário ou involuntário. $62 \%$ dos fissurados apresentaram algum hábito deletério, foram eles: respiração bucal, morder objetos, ranger os dentes, usar chupeta e roer as unhas. Não houve relatos de avaliados com hábito de chupar dedo. Por fim, 38\% dos fissurados avaliados não apresentou nenhum hábito deletério. Além disso, a ocorrência desses hábitos parafuncionais em pessoas com FLP é difícil generalizar devido a escassez de estudos, entretanto indivíduos com FLP apresentam consideravelmente mais anomalias e problemas orofaciais em relação a não fissurados (ALIGHIERI C, 2019).

Neste estudo, o maior destaque dos hábitos parafunfionais foi para o hábito de ranger os dentes, também conhecido como bruxismo. O bruxismo é caracterizado pela atividade noturna e/ou diurna involuntária dos músculos mastigatórios, rítmica ou espasmódica, podendo apresentar apertamento e/ou ranger dos dentes. Esse hábito parafuncional pode influenciar significativamente a qualidade de vida, acarretando em danos ao sistema mastigatório e desordens temporomandibulares, além de dores musculares e de cabeça, desgastes dentários, prejuízo aos tecidos periodontais, problemas respiratórios, distúrbios do sono e prejuízos na fala (SILVA SM, et al., 2018; RIOS LT, et al., 2018).

Os outros hábitos parafuncionais, respiração bucal, morder objetos, usar chupeta e roer as unhas, foram relatados, porém com menor frequência. Entretanto eles podem comprometer ainda mais a saúde do fissurado. A respiração bucal crônica durante a fase de crescimento pode influenciar negativamente 0 crescimento e desenvolvimento craniofacial alterando a morfologia maxilar, mandibular, altura facial, arcos dentários e funções labial e lingual (ALVES BR, et al., 2019).

Estes hábitos parafuncionais, denominados deletérios, envolvendo hábitos de mastigação sem fins nutritivos, como ranger e apertar dos dentes (bruxismo), onicofagia (roer unhas), morder objetos (canetas, roupas) ou sucção digital (chupar dedo). Estão associados a componentes emocionais, normalmente de etiologia multifatorial devendo ser desestimulados pelo profissional, pois interferem no crescimento esquelético normal dos maxilares, gerando danos e sobrecarga ao sistema estomatognático, como dores, desconforto, dificuldades na mastigação, perda do controle voluntário dos movimentos mandibulares, deslocamento articular e restrição dos movimentos (BARSI PC, et al., 2013; RAMOS HN e LIMA LB, 2017).

As pessoas fissuradas estão mais sujeitas a infecções oportunistas devido a comunicação buco-nasal, além disso restos de alimentos podem propiciar um novo foco de infecção. Então, antes e depois da alimentação é recomendado fazer a higiene oronasal com cotonete em água fervida para retirar resíduos de leite e evitar infecções (MONE F, et al., 2020). Essa maior possibilidade de infecções em pessoas fissuradas pode ser evidenciada pelos dados obtidos neste estudo em relação a internação nos últimos 5 anos. Dos fissurados avaliados, $83 \%$ relatou que esteve em condição de internação nos últimos 5 anos, apenas 17\% não esteve internado nesse período.

Atualmente, o protocolo de tratamento mais utilizado é o fechamento do lábio de forma cirúrgica com 3 meses de idade ou quando atinge o peso ideal para realização do procedimento. Já o reparo do palato, é feito em tempo único, com 1 ano de idade (ANDRADE CA, et al., 2019; MONE F, et al., 2020). Um dos objetivos dos reparos cirúrgicos das fissuras é separar as cavidades bucal e nasal, possibilitando boa funcionalidade na fonação e alimentação (ROCHA CL, et al., 2018).

Neste estudo em questão a situação cirúrgica reparadora de lábio e palato há uma minoria que ainda não passou por esses procedimentos no tempo ideal estabelecidos nos protocolos de tratamento atuais. Além disso, no presente estudo as crianças de até três anos de idade representam quase $60 \%$ dos participantes analisados, então o período de internação dos últimos 3 anos para a maioria compreende o período de internação para a realização das cirurgias reparadoras de lábios e palato.

Logo ao nascer, a pessoa com FLP deve ser submetida a uma avaliação, para que se identifique o tipo de fissura e sua extensão, para que seja traçado um plano de tratamento. Assim, a assistência odontológica a pacientes fissurados deve iniciar de forma precoce e envolver uma equipe multidisciplinar, com a finalidade de proporcionar suporte a todas as necessidades desse paciente (ALVES BR, et al., 2019). 


\section{CONCLUSÃO}

De acordo com os resultados, o sexo masculino é o de maior prevalência. A ocorrência de cárie aumentou junto com o avançar da idade. A fissura transforame prevaleceu em relação a pré-forame e pós-forame. $O$ bruxismo teve um maior destaque entre os hábitos deletérios. Por fim, pôde-se notar que uma grande maioria esteve internada nos últimos 5 anos e quase todos realizaram as cirurgias reparadoras no tempo certo. Os dados obtidos mostram condições satisfatórias de saúde bucal das pessoas com FL analisadas, entretanto a presença de hábitos parafuncionais é um fator que pode prejudicar todo o sistema estomatognático. Logo, conhecer o perfil do paciente fissurado é essencial para que sejam estabelecidos protocolos de tratamentos precocemente. Como também, os dados obtidos neste estudo poderão auxiliar novas pesquisas e contribuir para a caracterização de fissurados no Estado do Pará.

\section{AGRADECIMENTOS}

Apresentamos grande satisfação em poder desenvolver a pesquisa na Associação de Apoio ao Fissura do Labiopalatal Sorrisos Largos, nosso sincero agradecimento à então diretora da instituição Thatyana Mota. Além disso, demonstramos gratidão ao Centro de Especialidade Médicas e Odontológicas e sua então diretora Ludimila Magalhães Rodrigues da cunha.

\section{REFERÊNCIAS}

1. ALIGHIERI C, et al. Oral habits in children with Cleft (lip and) palate: A pilot study. Folia Phoniatrica et Logopaedica. 2019.

2. ALVES BR, et al. A importância de um protocolo preventivo no atendimento odontológico de pacientes fissurados: uma revisão sistemática da literatura. Arquivos em Odontologia, 2019.

3. AMORIM SM, et al. A Prática do aleitamento materno em crianças com fissuras labiopalatinas. Revista Eletrônica Acervo Saúde. 2019; 11: 296-296.

4. ANDRADE CA, et al. A Importância da equipe multiprofissional para a recuperação da criança com fenda labiopalatina. Revista Enfermagem Atual In Derme, 2019.

5. BARSI PC, et al. Prevalence of oral habits in children with cleft lip and palate. Plastic Surgery International. 2013.

6. COSTA VCR, et al. Aspectos etiológicos e clínicos das fissuras labiopalatinas. Revista de Medicina e Saúde de Brasília, 2019; 7: 2.

7. SILVA BJ, et al. Dificuldades enfrentadas por crianças com fissura labial e/ou palatina. Caderno de GraduaçãoCiências Biológicas e da Saúde, 2018; 5:1-67.

8. SILVA SM, et al. Perfil das mães com filhos diagnosticados com fissura labiopalatal. Revista Odontológica do Brasil Central, 2018; 27: 81.

9. SILVA YC, et al. Uma abordagem sobre a importância da atuação do profissional de nutrição no tratamento de crianças com fissuras labiopalatinas. Revista Eletrônica da Estácio Recife, 2018; 4:1.

10. BERNARDO B, et al. Fissuras lábio-palatinas: tipos de tratamento-revisão de literatura. Conversas Interdisciplinares, 2017; 13:3.

11. SANTOS RC, et al. A Importância da fonoaudiologia e ortodontia no tratamento de pacientes com fissura labioplatina: Uma revisão de literatura. Scientific-Clinical Odontology, 2019.

12. KUHN VD, et al. Fissuras labiopalatais: revisão da literatura. Disciplinarum Sciential Saúde, 2016; 2: $237-245$.

13. LACERDA RH, et al. Prevalência de defeitos de esmalte em indivíduos portadores de fissuras labiopalatinas da Paraíba, Brasil. Rev Cubana Estomatol, 2012; 49:11-9.

14. MONE F, et al. Prevalência de fendas orofaciais em bebês e fatores que interferem na amamentação. Enfermagem Brasil, 2020; 3: 180-185.

15. NUNES VH; PEROSA GB. Cárie dentária em crianças de 5 anos: fatores sociodemográficos, lócus de controle e atitudes parentais. Ciência \& Saúde Coletiva, 2017; 22:191-200.

16. OLIVEIRA MF, BANDEIRA MB. Procedimento terapêutico multiprofissional de pacientes com fissura labiopalatal: relato de experiência. Academus Revista Científica da Saúde, 2018. 1:22-28.

17. PESSOA EA, et al. Enxertos ósseos alveolares na fissura labiopalatina: protocolos atuais e perspectivas futuras. Revista de Odontologia da Universidade Cidade de São Paulo, 2017; 27: 49-55.

18. PIRES AC, et al. Desenvolvimento dental e idade cronológica em pacientes com fissuras labiopalatinas: uma revisão de literatura. Archives of health investigation, 2020; 8:9.

19. RAMOS HN, LIMA LB. Relação do bruxismo com hábitos deletérios infantis em crianças de 4 a 7 anos. Artigo Científico (Trabalho de Conclusão de Curso)-Universidade São Lucas, Porto Velho, 2017.

20. RIOS LT, et al. Bruxismo infantil e sua associação com fatores psicológicos-revisão sistemática da literatura. Revista de Odontologia da Universidade Cidade de São Paulo, 2018; 30: 64-76.

21. ROCHA CL. Perfil de saúde bucal do paciente portador de fissura labiopalatina: estudo epidemiológico em hospital pediátrico do Nordeste Brasileiro. Monografia (Trabalho de Conclusão de Curso)-Universidade Federal do Ceará, Fortaleza, 2018.

22. SILVA FC, et al. Impact of temporomandibular disorders and sleep bruxism on oral health-related quality of life of individuals with complete cleft lip and palate. J Craniofac Surg, 2018; 6:1505-1508.

23. SHIBUKAWA BM, et al. Factors associated with the presence of cleft lip and/or cleft palate in Brazilian newborns. Revista Brasileira de Saúde Materno Infantil, 2019; 19: 947-956. 